\title{
Intraoperative neurophysiological monitoring in vestibular schwannoma surgery: advances and clinical implications
}

\author{
A. Samy Youssef, M.D., Ph.D., ${ }^{1}$ and Angela E. Downes, M.D. ${ }^{2}$ \\ ${ }^{1}$ Department of Neurosurgery, University of South Florida, Tampa, Florida; \\ and ${ }^{2}$ Department of Neurosurgery, University of Mississippi, Jackson, Mississippi
}

\begin{abstract}
Object. Intraoperative neurophysiological monitoring has become an integral part of vestibular schwannoma surgery. The aim of this article was to review the different techniques of intraoperative neurophysiological monitoring in vestibular schwannoma surgery, identify the clinical impact of certain pathognomonic patterns on postoperative outcomes of facial nerve function and hearing preservation, and highlight the role of postoperative medications in improving delayed cranial nerve dysfunction in the different reported series.

Methods. The authors performed a review of the literature regarding intraoperative monitoring in acoustic/ vestibular schwannoma surgery. The different clinical series representing different monitoring techniques were reviewed. All the data from clinical series were analyzed in a comprehensive and comparative model.

Results. Intraoperative brainstem auditory evoked potential monitoring, direct cochlear nerve action potential monitoring, and facial nerve electromyography are the main tools used to assess the functional integrity of an anatomically intact cranial nerve. The identification of pathognomonic brainstem auditory evoked potential and electromyography patterns has been correlated with postoperative functional outcome. Recently, perioperative administration of intravenous hydroxyethyl starch and nimodipine as vasoactive and neuroprotective agents was shown to improve vestibular schwannoma functional outcome in few reported studies.

Conclusions. Recent advances in electrophysiological technology have considerably contributed to improvement in functional outcome of vestibular neuroma surgery in terms of hearing preservation and facial nerve paresis. Perioperative intravenous nimodipine and hydroxyethyl starch may be valuable additions to surgery.
\end{abstract}

(DOI: 10.3171/2009.8.FOCUS09144)

\section{KeY WoRds • vestibular schwannoma - neurophysiological monitoring • facial nerve function $\bullet \quad$ hearing preservation}

I NTRAOPERATIVE neurophysiological monitoring has played an important role in modern skull base surgery and vestibular schwannoma surgery. Facial nerve EMG not only helps with identifying the nerve anatomically, but can also preserve the functional integrity and helps to predict the postoperative functional outcome of the nerve. The introduction of routine intraoperative facial nerve monitoring has significantly reduced the incidence of facial nerve paresis in a postoperative physically intact nerve. ${ }^{10,23,24,26}$ Brainstem auditory evoked potentials are widely used for intraoperative monitoring of cochlear nerve and brainstem function as a means of hearing preservation during vestibular schwannoma surgery. The different patterns of BAEP have been identified and correlated with postoperative hearing outcome. ${ }^{13,20}$ Direct

Abbreviations used in this paper: $\mathrm{BAEP}=$ brainstem auditory evoked potential; $\mathrm{CN}=$ cranial nerve $; \mathrm{CNAP}=$ cochlear nerve action potential; ECOG = electrocochleography; EMG = electromyography. intraoperative cochlear nerve monitoring is a relatively new tool that has been applied for hearing preservation during the past decade; however, its role is still controversial. ${ }^{18,22}$ Recently, identification of certain intraoperative pathognomonic electrophysiological patterns has translated into perioperative vasoactive and neuroprotective pharmacotherapy with calcium channel blockers in an attempt to decrease delayed hearing loss and facial paresis. ${ }^{31,32,34}$ In this article, we review the different techniques of intraoperative neurophysiological monitoring in vestibular schwannoma surgery, identify the clinical impact of certain patterns on postoperative outcome of facial nerve function and hearing preservation, and highlight the role of postoperative medications in improving delayed $\mathrm{CN}$ dysfunction in the different reported series.

\section{Methods}

We performed a MEDLINE search on PubMed for acoustic/vestibular schwannoma surgery with a result of 3507 articles. A refined search for intraoperative moni- 

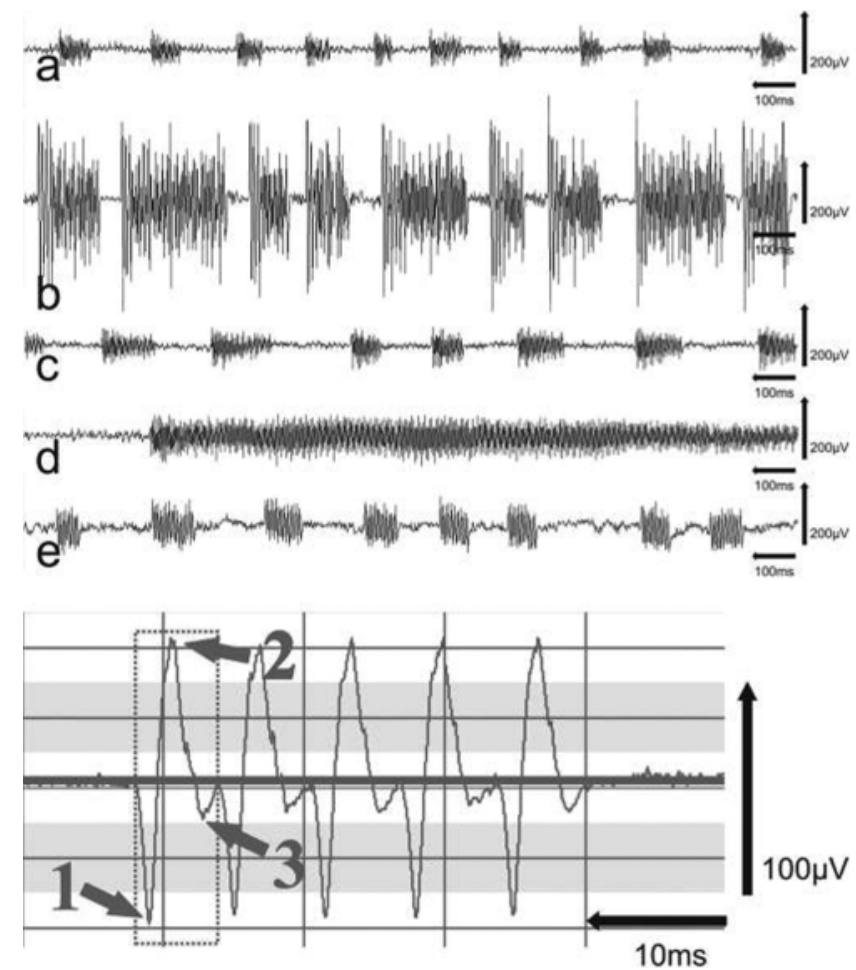

FIG. 1. Upper: Tracings labeled "a" through "e" show a variety of $A$ trains demonstrating differences in amplitude, frequency, length, and overall appearance of the patterns. The example labeled "d" shows a single, prolonged $A$ train with sudden onset and a high frequency (200 $\mathrm{Hz}$ ). This A train also shows an amplitude decrescendo. In contrast to the "d" tracing, all other examples consist of short, repetitive A trains, which is very typical for the pattern. Example " $b$ " shows A trains of comparatively high amplitude, in the range of $400 \mu \mathrm{V}$. In examples "a" and "C", amplitude decrescendo is seen within the single A trains. Lower: $A$ very short $A$ train with significant spread on the time axis is shown to demonstrate the general characteristics of the pattern. This A train is composed of 5 triphasic elements (maximum and minimum amplitudes for the first element are marked by numbered arrows). The elements are of the same entity; they look alike. The time intervals between the single elements show very little variation, forming a stable frequency of $105 \mathrm{~Hz}$. Reprinted with permission from Prell $\mathbf{J}$ et al: $\mathbf{J}$ Neurosurg 106:826-832, 2007.

toring in acoustic/vestibular schwannoma surgery resulted in 288 articles; 249 were in the English language. The systematic review led to 2 major categories: intraoperative facial nerve monitoring and BAEPs for hearing preservation. The various clinical series representing different monitoring techniques were reviewed. Recent studies (1990-present) with the largest patient series (between 30 and 150 patients) that focused on the modern neurophysiological monitoring techniques and their clinical impact were selected. The end result was 34 main articles in the English language. All data from clinical series were analyzed in a comprehensive and comparative model.

\section{Results}

Intraoperative EMG Monitoring of Facial Nerves

Recording Procedure. Induction of anesthesia is performed with avoidance of inhalational agents and neuro-

\section{T. $\varnothing=14 \mathrm{~mm}$, PTA=24 dB, SDS=100\%}

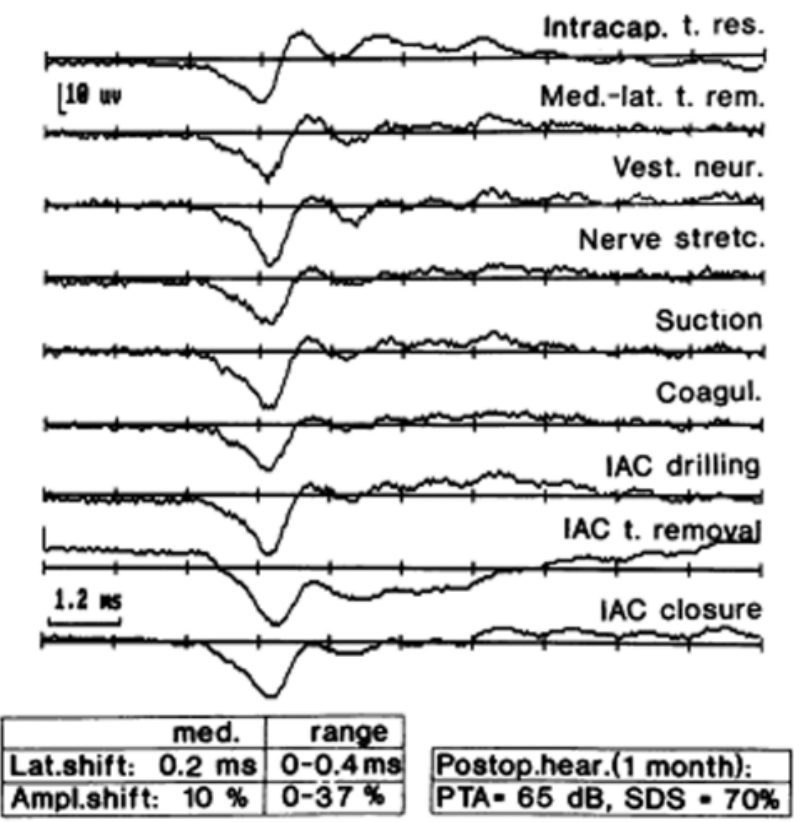

FIG. 2. Cochlear nerve action potentials recorded at different surgical steps during removal of an acoustic neuroma. Ampl. = amplitude; coagul. = coagulation; hear. = hearing; $I A C=$ internal auditory canal; intracap. t. res. $=$ intracapsular tumor resection; Med.-lat. t. rem. $=$ medial to lateral tumor removal; PTA = pure-tone average; $S D S=$ speech discrimination score; stretc. = stretching; Vest. neur. = vestibular neurectomy. Reprinted from Colletti V, Fiorino FG, Carner M, et al: Intraoperative monitoring for hearing preservation and restoration in acoustic neuroma surgery. Skull Base Surg 10:187-195, 2000 with permission of the publisher.

muscular blockers. Hemodynamic stability is maintained. Parallel pairs of noninsulated needle electrodes are placed transcutaneously in the lateral orbiculus oculi, the nasal muscle, and the orbicularis oris. The facial nerve stimulation probe is used for direct electrical stimulation to localize the nerve prior to tumor resection and intermittently during dissection of tumor from the nerve. After resection of the vestibular schwannoma, the probe is used to elicit the stimulation threshold at the brainstem and medial to the tumor dissection. The lowest stimulation threshold can be elicited by first applying $0.05 \mathrm{~mA}$ and increased in $0.05-\mathrm{mA}$ increments until response amplitude is obtained. The response amplitude achievable with the lowest stimulation threshold is recorded and can be used as a predictor of postoperative facial nerve function. ${ }^{3,7,15,21,33}$

Electromyography Patterns. In addition to intermittent direct electrical stimulation, continuous loudspeaker monitoring is applied as a method of real-time assessment of the functional integrity of the facial nerve. In loudspeaker monitoring, neurotonic activity in the form of audible prolonged activity reflects functional damage to the nerve. This pattern is called train activity. Several train patterns can be recorded with respect to wave characteristics, frequencies, and amplitudes (Fig. 1). The A train, described as sinusoidal pattern of high frequency 
and homogeneous appearance, was shown to be the most sensitive and specific pattern that can indicate postoperative paresis. ${ }^{26}$ It produces high-frequency sound of up to $210 \mathrm{~Hz}$ from the loud speaker with maximum amplitudes ranging from $100-200 \mu \mathrm{V}$ and never exceeds $500 \mu \mathrm{V}$, whereas the duration varies between milliseconds and several seconds. ${ }^{24}$

\section{Intraoperative Cochlear Nerve Monitoring}

Cochlear nerve function monitoring for the purpose of hearing preservation can be performed by recording auditory evoked responses from the ear canal and scalp electrodes, or by direct intraoperative cochlear nerve monitoring. A hearing preservation approach (middle fossa or retrosigmoid transmeatal) is chosen.

\section{Brainstem Auditory Evoked Potentials}

The BAEPs are recorded in response to stimulation delivered to the ipsilateral ear. In large tumors causing brainstem compression with no serviceable ipsilateral hearing, BAEPs may be recorded from the contralateral ear as a measure of brainstem function. The BAEP patterns are generally resistant to sedation and general anesthesia. After induction of general anesthesia, a soft ear mold attached with a 12-inch plastic tube is placed and sealed inside the ear canal. For fast auditory brainstem response, a needle connected to the positive grid of the differential amplifier is inserted transcutaneously at the vertex, and a ground electrode is placed $3 \mathrm{~cm}$ anterior to the vertex. A needle connected to the negative grid of the differential amplifier is placed in the pretragal area or mastoid tip area. A brief click or tone is delivered at an intensity level of 90-100 dB and at a rate of 31-51 pulses per second. Baseline responses for each ear are recorded before the beginning of surgery and are used as baselines throughout the case. The classic BAEP comprises 5-7 peaks, all occurring within $10 \mathrm{msec}$ of the click; the first 5 peaks (Waves $\mathrm{I}-\mathrm{V}$ ) are the main peaks used in clinical practice (see Fig. 3 in the paper by Legatt ${ }^{14}$ ). Waves IV and $\mathrm{V}$ are generated at the upper pons and lower midbrain. ${ }^{4}$ Wave $\mathrm{V}$ tends to be the most robust and is the most closely monitored during surgery. The surgeon is alerted when the change in latency of Wave $\mathrm{V}$ exceeds $0.5 \mathrm{msec}$ or if there is a change in or disappearance of any wave. ${ }^{14}$

\section{Direct CNAPS}

The CNAP is a tool used to evaluate the cochlear nerve function in real time. It is used in several centers in addition to BAEPs only during a retrosigmoid approach. The recording can be performed after exposure of the cochlear portion of the CN VIII located on the caudal side of the nerve near the brainstem and anteroventral to the $\mathrm{CN}$ VIII at the porus acusticus (Fig. 2). A Teflon-coated multistrand silver wire electrode with a cotton pledget attached to the wire is placed directly on the cochlear nerve proximal to the tumor. The cotton pledget is secured with the help of Gelfoam to maintain contact with the nerve. ${ }^{18}$ The negative electrode is placed at the contralateral mastoid tip area, and the ground electrode is placed at the vertex. The stimuli used are similar to those used in BAEP but with a smaller number of summations. The surgeon is alerted to any change in the CNAP's wave patterns (Fig. 2).

Intraoperative ECOG is another near-field technique that has been advocated by some neurootologists to directly monitor the cochlear nerve. ${ }^{13,35}$ Electrocochleography monitoring generally requires transtympanic electrode placement because electrode placement in the external ear does not yield a large enough amplitude. ${ }^{35} \mathrm{~A}$ perforation is made in the tympanic membrane for cotton wick or needle electrode placement. Electrode placement outside the surgical field is a major advantage of ECOG over CNAP.

\section{Discussion}

Recent advances in intraoperative neurophysiological monitoring techniques have made preservation of $\mathrm{CN}$ function a definitive goal in skull base surgery. Resection of cerebellopontine angle lesions, in particular vestibular schwannoma, has benefitted the most from facial and cochlear nerve monitoring..$^{10,11,28}$ Facial nerve paresis significantly impairs the quality of life and may require several cosmetic and palliative procedures. Postoperative hearing loss in a previously hearing ear leaves the patient with some sort of a permanent handicap and is perceived as a setback for microsurgery. With the refinement of microsurgical techniques and surgeons accumulating more experience with vestibular schwannoma surgery, the goals of surgery are set at a higher level. The early collaboration of neurootologists and neurosurgeons in skull base surgery blossomed the introduction of intraoperative neurophysiological monitoring to vestibular schwannoma surgery, which significantly increased the likelihood of successful preservation of facial and cochlear nerve function. Samii and Matthies ${ }^{29}$ reported that functional hearing preservation was possible in $39-50 \%$ of 1000 patients after surgery. In other series, hearing preservation was possible in $57 \%$ and facial nerve preservation was possible in $95-100 \%$ of all patients with tumors $<1 \mathrm{~cm}$; hearing preservation was possible in $33 \%$ and facial nerve preservation was possible in $80-92 \%$ of patients with tumors measuring 1-2 cm; and hearing preservation was possible in $6 \%$ and facial preservation was possible in $50-76 \%$ of patients with tumors $>2 \mathrm{~cm}^{30}$

\section{Facial Nerve Monitoring and Functional Preservation}

Since Prass and Lüders ${ }^{23}$ introduced the direct electrical stimulation and loud-speaker monitoring, it has become the gold standard for intraoperative facial nerve monitoring. The facial nerve position is relatively fixed in the internal auditory meatus and pons, and it can be best identified after drilling the posterior wall of the meatus. The Prass probe is used to identify the nerve in the canal at the beginning of tumor dissection. Intermittent stimulation is an invaluable tool in tracking the facial nerve throughout the dissection of the tumor capsule and vestibular nerve from the facial nerve. Stimulation is performed once again before cutting the vestibular nerve at the brainstem at the final stage of tumor eradication. The vestibular nerve is identified by its inability to evoke facial muscle activity, in contrast to the facial nerve. 
A. S. Youssef and A. E. Downes

TABLE 1: A sample of studies comparing direct CNAPs and BAEPs in hearing preservation for vestibular schwannoma surgery

\begin{tabular}{lcccccc}
\hline & \multicolumn{3}{c}{ No. of Ops } & & \\
\cline { 2 - 4 } \multicolumn{1}{c}{ Authors \& Year } & Total & W/BAEPs & W/CNAPs & $\begin{array}{c}\text { BAEP Service- } \\
\text { able Hearing (\%) }\end{array}$ & $\begin{array}{c}\text { CNAP Service- } \\
\text { able Hearing (\%) }\end{array}$ & Tumor size (cm) \\
\hline Danner et al., 2004 & 86 & 22 & 44 & 27 & 43 & $\leq 1$ to $\geq 2.5$ \\
Piccirillo et al., 2008 & 99 & 24 & 75 & 20.8 & 26.7 & $\leq 1.5$ \\
Nedzelski et al., 1994* & 80 & & 56 & & 38 & small \\
Colletti et al., 2000 & 158 & 14 & $92 \dagger$ & 30 & $70 \dagger$ & small \\
\hline * The CANPs were compared with historical controls for BAEPs. & & & \\
+ The CANP and BAEP monitoring results were combined.
\end{tabular}

\section{Prediction of Postoperative Facial Nerve Outcome}

The facial nerve can be anatomically preserved in most cases; however, 20-70\% of patients suffer from postoperative facial nerve functional deficits. The loudspeaker monitoring has been introduced as a continuous monitoring tool that provides real-time updates on the functional integrity of the nerve. It was concluded that all prolonged audible activity in the EMG patterns recorded from facial muscles indicates functional damage to the nerve. ${ }^{10,23}$ Recently, the A train has clearly been identified as the only intraoperative EMG pattern corresponding to deterioration of facial nerve function. ${ }^{26}$ Driven by perfection in vestibular schwannoma surgery, several studies have investigated the correlation of intraoperative facial EMG monitoring parameters and their ability to predict facial nerve outcomes. Several EMG parameters were studied: the A-train neurotonic discharges, stimulus threshold, response amplitude, and proximal to distal amplitude ratio. Prell et al. ${ }^{24}$ found a strong correlation between the length of train time and deterioration of postoperative facial nerve function. Patients with no preoperative facial paresis were found to be able to tolerate up to 0.5 seconds of train time. At up to 10 seconds of train time, postoperative impairment was limited to deterioration by 1 House-Brackmann grade. ${ }^{12}$ Also the ability of stimulus threshold and tumor size to predict postoperative facial nerve function has been evaluated. Fenton et al. ${ }^{7}$ showed that a stimulus threshold $<0.05 \mathrm{~mA}$ and a smaller tumor size correlated with a House-Brackmann Grade of III or less in $88 \%$ of patients (59 of 67) initially after surgery. Neff et al. ${ }^{21}$ found that an intraoperative EMG stimulus threshold of $0.05 \mathrm{~mA}$ or less and a response amplitude of $240 \mu \mathrm{V}$ or more can help predict a House-Brackmann Grade I or II facial nerve function with a $98 \%$ probability. Mandpe et al. ${ }^{15}$ showed that a stimulus threshold of 0.1 or less combined with a response amplitude of $200 \mu \mathrm{V}$ or greater predicted a House-Brackmann Grade I-II facial nerve outcome in $88 \%$ of 44 patients. Goldbrunner et al. ${ }^{8}$ demonstrated that a ratio of response amplitude recorded from stimulating the nerve both proximal and distal to the tumor dissection of 0.1 or less correlated with a poor facial functional outcome in $75 \%$ of patients at 6 months.

\section{Cochlear Nerve Monitoring and Hearing Preservation}

Hearing preservation is defined as preservation of hearing within normal and social hearing classification, that is, pure tone audiometry $\leq 50 \mathrm{~dB}$ and speech discrimination score $\geq 50 \%$ according to guidelines of the American Academy of Otolaryngology-Head and Neck surgery. ${ }^{1}$ The surgical approach used is either the middle fossa approach or retrosigmoid transmeatal approach. Intraoperative cochlear nerve monitoring has been traditionally performed with BAEPs. However, direct CNAP monitoring has been used in multiple centers and is theoretically the most ideal technique since surgical manipulations resulting in reduced activity or desynchronization of high-frequency nerve fibers can be detected as a change in amplitude or latency of the wave. The classic BAEP comprises 5-7 peaks. Wave $\mathrm{V}$ tends to be the most robust and is the most closely monitored during surgery. The surgeon is alerted when the change in latency of Wave $\mathrm{V}$ exceeds $0.5 \mathrm{msec}$ or if there is change or disappearance of any wave. Several studies have failed to show strong evidence of higher hearing preservation with intraoperative cochlear nerve monitoring. The main benefit is the ability to predict outcome in those patients who have diminished responses at the end of surgery. ${ }^{13,20}$ Both CNAP and ECOG have the advantage of being near-field techniques in which electrodes are placed close to the $\mathrm{CN}$ VIII or cochlea. Larger amplitude signals are produced and acquisition takes only $2-3$ seconds (in contrast to minutes with BAEP), thus allowing near real-time feedback to the surgeon. Several studies have reported that CNAP monitoring and ECOG are more reliable than BAEP monitoring, while others have failed to prove their superiority in terms of hearing preservation (Table 1). ${ }^{6,18,19,20,22,25,27}$

\section{Outcome Prediction and Improvement}

Ambitions to improve vestibular schwannoma surgery were beyond simply prediction of procedural outcome. The introduction of intraoperative monitoring has taken vestibular schwannoma surgery from impressionism to realism. Recognition of intraoperative $\mathrm{CN}$ functional damage did not stop at just warning the surgeon to stop dissecting and manipulating the facial or cochlear nerves. Perioperative steroids have been routinely used to minimize postoperative nerve damage attributed to postoperative edema. The modest effect of steroids in improving postoperative facial and cochlear nerve function drove tremendous research efforts focused on improving micro- 
circulation in anatomically preserved nerves. Animal studies have shown the neuroprotective effect of the calcium channel blocker nimodipine as it improves resprouting, promotes axonal growth and remyelination, and reduces polyneuronal innervations of the target muscles. . $^{2,9,16,17,32}$ Clinical trials based on medicating patients who develop reversible pathognomonic intraoperative BAEP patterns or A-train EMG activity with a combination of intravenous HES, a vasoactive agent, and nimodipine have shown improvement in long-term functional results. ${ }^{31,32,34}$ In a recent important prospective and open-label randomized pilot study reported by Scheller et al. ${ }^{30}$ prophylactic vasoactive treatment consisting of nimodipine and HES showed significantly better results concerning preservation of facial and cochlear nerve function in vestibular schwannoma surgery. This study also indicated that prophylactic use combined with surgery seems to be more successful in hearing preservation and facial functional recovery than intraoperative application.

\section{Conclusions}

Successful preservation of facial and cochlear function in vestibular schwannoma surgery has been improved by the introduction of intraoperative neurophysiological monitoring techniques. Intraoperative BAEPs, direct CNAPs, and facial nerve EMG are the main tools to assess the functional integrity of an anatomically intact $\mathrm{CN}$. The identification of pathognomonic BAEP and EMG patterns has been correlated with postoperative functional outcomes. Experimental and clinical studies concerning cochlear and facial nerve function have set the stage for the perioperative administration of intravenous HES and nimodipine as vasoactive and neuroprotective agents that may improve vestibular schwannoma functional outcome.

\section{Disclaimer}

The authors report no conflict of interest concerning the materials or methods used in this study or the findings specified in this paper.

\section{References}

1. Angelov DN, Neiss WF, Streppel M, Andermahr J, Mader K, Stennert E: Nimodipine accelerates axonal sprouting after surgical repair of rat facial nerve. J Neurosci 16:1041-1048, 1996

2. Axon PR, Ramsden RT: Assessment of real-time clinical facial function during vestibular schwannoma resection. Laryngoscope 110:1911-1915, 2000

3. Buchwald JS, Huang C: Far-field acoustic response: origins in the cat. Science 189:382-384, 1975

4. Colletti V, Bricolo A, Fiorino FG, Bruni L: Changes in directly recorded cochlear nerve compound action potentials during acoustic tumor surgery. Skull Base Surg 4:1-9, 1994

5. Colletti V, Fiorino FG, Carner M, Cumer G, Giarbini N, Sacchetto L: Intraoperative monitoring for hearing preservation and restoration in acoustic neuroma surgery. Skull Base Surg 10:187-195, 2000

6. Danner C, Mastrodimos B, Cueva RA: A comparison of direct eighth nerve monitoring and auditory brainstem response in hearing preservation surgery for vestibular schwannoma. Otol Neurotol 25:826-832, 2004
7. Fenton JE, Chin RY, Fagan PA, Sterkers O, Sterkers JM: Predictive factors of long-term facial nerve function after vestibular schwannoma surgery. Otol Neurotol 23:388-392, 2002

8. Goldbrunner RH, Schlake HP, Milewski C, Tonn JC, Helms J, Roosen K: Quantitative parameters of intraoperative electromyography predict facial nerve outcomes for vestibular schwannoma surgery. Neurosurgery 46:1140-1148, 2000

9. Guntinas-Lichius O, Martinez-Portillo F, Lebek J, Angelov DN, Stennert E, Neiss WF: Nimodipine maintains in vivo the increase in GFAP and enhances the astroglial ensheathment of surviving motoneurons in the rat following permanent target deprivation. J Neurocytol 26:241-248, 1997

10. Harner SG, Daube JR, Ebersold MJ, Beatty CW: Improved preservation of facial nerve function with use of electrical monitoring during removal of acoustic neuromas. Mayo Clin Proc 62:92-102, 1987

11. Harper CM, Harner SG, Slavit DH, Litchy WJ, Daube JR, Beatty $\mathrm{CW}$, et al: Effect of BAEP monitoring on hearing preservation during acoustic neuroma resection. Neurology 42:1551-1553, 1992

12. House JW, Brackmann DE: Facial nerve grading system. Otolaryngol Head Neck Surg 93:146-147, 1985

13. Jackson LE, Roberson JB Jr: Acoustic neuroma surgery: use of cochlear nerve action potential monitoring for hearing preservation. Am J Otol 21:249-259, 2000

14. Legatt AD: Mechanisms of intraoperative brainstem auditory evoked potential changes. J Clin Neurophysiol 19:396-408, 2002

15. Mandpe AH, Mikulec A, Jackler RK, Pitts LH, Yingling CD: Comparison of response amplitude versus stimulation threshold in predicting early postoperative facial nerve function after acoustic neuroma resection. Am J Otol 19:112-117, 1998

16. Mattsson P, Aldskogius H, Svensson M: Nimodipine-induced improved survival rate of facial motor neurons following intracranial transection of the facial nerve in the adult rat. $\mathbf{J}$ Neurosurg 90:760-765, 1999

17. Mattsson P, Janson AM, Aldskogius H, Svensson M: Nimodipine promotes regeneration and functional recovery after intracranial facial nerve crush. J Comp Neurol 437:106-117, 2001

18. Moller AR, Jannetta PJ: Auditory evoked potentials recorded from the cochlear nucleus and its vicinity in man. J Neurosurg 59:1013-1018, 1983

19. Monsell EM, Balkany TA, Gates GA, Goldenberg RA, Meyerhoff WL, House JW: Committee on Hearing and Equilibrium guidelines for the evaluation of hearing preservation in acoustic neuroma (vestibular schwannoma). Otolaryngol Head Neck Surg 113:179-180, 1995

20. Nedzelski JM, Chiong CM, Cashman MZ, Stanton SG, Rowed DW: Hearing preservation in acoustic neuroma surgery: value of monitoring cochlear nerve action potentials. Otolaryngol Head Neck Surg 111:703-709, 1994

21. Neff BA, Ting J, Dickinson SL, Welling DB: Facial nerve monitoring parameters as a predictor of postoperative facial nerve outcomes after vestibular schwannoma resection. Otol Neurotol 26:728-732, 2005

22. Piccirillo E, Hiraumi H, Hamada M, Russo A, De Stefano A, Sanna M: Intraoperative cochlear nerve monitoring in vestibular schwannoma surgery-does it really affect hearing outcome? Audiol Neurootol 13:58-64, 2008

23. Prass RL, Lüders H: Acoustic (loudspeaker) facial electromyographic monitoring: Part 1. Evoked electromyographic activity during acoustic neuroma resection. Neurosurgery 19:392-400, 1986

24. Prell J, Rampp S, Romstock J, Fahlbusch R, Strauss C: Train time as a quantitative electromyographic parameter for facial nerve function in patients undergoing surgery for vestibular schwannoma. J Neurosurg 106:826-832, 2007

25. Roberson J, Senne A, Brackmann D, Hitselberger WE, Saun- 


\section{A. S. Youssef and A. E. Downes}

ders J: Direct cochlear nerve action potentials as an aid to hearing preservation in middle fossa acoustic neuroma resection. Am J Otol 17:653-657, 1996

26. Romstock J, Strauss C, Fahlbusch R: Continuous electromyography monitoring of motor cranial nerves during cerebellopontine angle surgery. J Neurosurg 93:586-593, 2000

27. Rowed DW, Nedzelski JM, Cashman MZ, Stanton S, Harrison $\mathrm{RV}$ : Cochlear nerve monitoring during cerebellopontine angle operations. Can J Neurol Sci 15:68-72, 1988

28. Samii M, Gerganov V, Samii A: Improved preservation of hearing and facial nerve function in vestibular schwannoma surgery via the retrosigmoid approach in a series of 200 patients. J Neurosurg 105:527-535, 2006

29. Samii M, Matthies C: Management of 1000 vestibular schwannomas (acoustic neuromas): the facial nerve-preservation and restitution of function. Neurosurgery 40:684-685, 1997

30. Scheller C, Richter HP, Engelhardt M, Koenig R, Antoniadis G: The influence of prophylactic vasoactive treatment on cochlear and facial nerve functions after vestibular schwannoma surgery: a prospective and open-label randomized pilot study. Neurosurgery 61:92-98, 2007

31. Scheller C, Strauss C, Fahlbusch R, Romstock J: Delayed facial nerve paresis following acoustic neuroma resection and postoperative vasoactive treatment. Zentralbl Neurochir 65:103-107, 2004
32. Sekiya T, Yagihashi A, Asano K, Suzuki S: Nimodipine ameliorates trauma-induced cochlear neuronal death. Neurol Res 24:775-780, 2002

33. Sobottka SB, Schackert G, May SA, Wiegleb M, Reiss G: Intraoperative facial nerve monitoring (IFNM) predicts facial nerve outcome after resection of vestibular schwannoma. Acta Neurochir (Wien) 140:235-243, 1998

34. Strauss C: The facial nerve in medial acoustic neuromas. $\mathbf{J}$ Neurosurg 97:1083-1090, 2002

35. Winzenburg SM, Margolis RH, Levine SC, Haines SJ, Fournier EM: Tympanic and transtympanic electrocochleography in acoustic neuroma and vestibular nerve section surgery. Am J Otol 14:63-69, 1993

Manuscript submitted June 14, 2009.

Accepted August 14, 2009.

Address correspondence to: A. Samy Youssef, M.D., Ph.D., Department of Neurosurgery, University of South Florida, 2 Tampa General Circle, Tampa, Florida 33606. email: ayoussef@ health.usf. edu. 